\begin{tabular}{|c|c|}
\hline Title & Rhodium-Catalyzed Enantioselective A rylation of A liphatic Imines \\
\hline Author(s) & Kato, Naoya; Shirai, Tomohiko; Y amamoto, Y asunori \\
\hline Citation & $\begin{array}{l}\text { Chemistry-A European journal, 22(23), } 7739-7742 \\
\text { https://doi.org/10.1002/chem.201601246 }\end{array}$ \\
\hline Issue Date & 2016-06-01 \\
\hline Doc URL & http:/hdl.handle.net/2115/65861 \\
\hline Rights & $\begin{array}{l}\text { This is the peer reviewed version of the following article: Chemistry A European journal, 22(23) June } 12016 \text { Pages } \\
7739-7742 \text {, which has been published in final form at } \\
\text { http://onli inelibrary. wiley. com /doi } 10.1002 \text { chem. } 201601246 / \text { abstract. This article may be used for non-commercial } \\
\text { purposes in accordance With Wiley-V CH Terms and Conditions for self-archiving. }\end{array}$ \\
\hline Type & article (author version) \\
\hline File Information & chem.201601246R1.pdf \\
\hline
\end{tabular}

Instructions for use 


\title{
Rhodium-Catalyzed Enantioselective Arylation of Aliphatic Imines
}

\author{
Naoya Kato, Tomohiko Shirai, and Yasunori Yamamoto*a] \\ Dedication ((optional))
}

\begin{abstract}
Chiral rhodium(I)-catalyzed highly enantioselective arylation of aliphatic $\mathrm{N}$-sulfonyl aldimines with arylboronic acids has been developed. This transformation is achieved by the use of a rhodium/bis(phosphoramidite) catalyst to give enantiomerically enriched $\alpha$-branched amines (up to $99 \%$ ee). In addition, this system enables efficient synthesis of (+)-NPS R-568 and Cinacalcet which are calcimimetic agents.
\end{abstract}

\section{Introduction}

As attractive structural elements, chiral $\alpha$-branched aryl alkyl amine derivatives are present in biologically active molecules and drugs such as NPS R-568, ${ }^{[1]}$ Cinacalcet,${ }^{[2]}$ and Maraviroc ${ }^{[3]}$ (Figure 1). Several strategies have been developed to access chiral $\alpha$-branched aryl alkyl amines in an enantioselective manner. ${ }^{[4]}$ Among those synthetic approaches, asymmetric arylation of aliphatic aldimines with arylboronic acids is a particularly useful and powerful synthetic method. Several groups have reported successful examples of catalytic asymmetric arylation of alkyl imines to give $\alpha$-branched amines with excellent levels of asymmetric induction. ${ }^{[5,6]}$

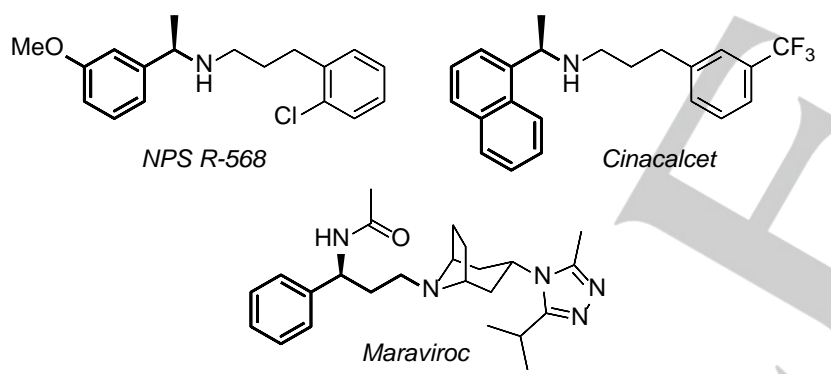

Figure 1. Examples of drugs having chiral amine parts.

For example, in 2011, Lin and co-workers reported a rhodium/diene-catalyzed enantioselective arylation of N-tosyl or -nosyl aliphatic aldimines. ${ }^{[5 c]}$ Recently, Beisel and Manolikakes reported a convenient enantioselective arylation of aromatic and aliphatic imines that were synthesized in situ from aldehydes and sulfonamides. ${ }^{[5 d]}$

We have succeeded in the development of a bis(phosphoramidite) ligand, called Me-BIPAM, that possesses a linked-binol backbone and shows a unique reactivity (Figure 2 ).

[a] N. Kato, T. Shirai, Prof. Dr. Y. Yamamoto

Division of Chemical Process Engineering and Frontier Chemistry Center (FCC), Faculty of Engineering, Hokkaido University Kita 13 Nishi 8 Kita-ku, Sapporo, Hokkaido 060-8628 (Japan) E-mail: yasuyama@eng.hokudai.ac.jp

Supporting information for this article is given via a link at the end of the document.
The properties of these ligands can be adjusted by changing the linker atom such as nitrogen, oxygen and sulfur. BIPAM ligands with a transition-metal complex work as an effective catalyst for asymmetric 1,2-addition ${ }^{[7]}$ and 1,4-addition reactions of arylboronic acids, ${ }^{[8]}$ hydrogenation ${ }^{[9]}$ and $\mathrm{C}-\mathrm{H}$ functionalization. ${ }^{[10]}$

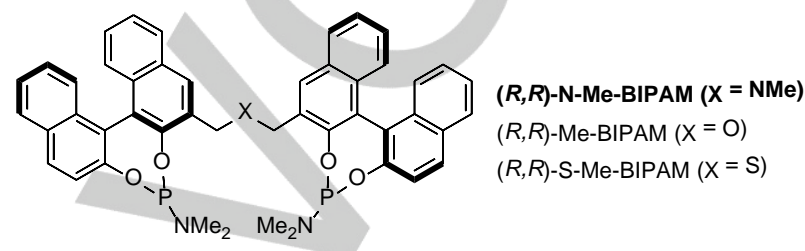

Figure 2. BIPAM ligands.

We have already reported enantioselective arylation of $\mathrm{N}$ sulfonyl aromatic aldimines ${ }^{[7 a]}$ and iminoesters ${ }^{[7 c]}$ with aryl boron reagents in the presence of a rhodium/bis(phosphoramidite) (NMe-BIPAM) catalyst. Herein, we report rhodium/N-Me-BIPAMcatalyzed arylation of aliphatic imines with arylboronic acids, which proceeds enantioselectively to give a variety of optically active $\alpha$-branched amines. Furthermore, we show that the newly developed reaction can be used for the synthesis of NPS R-568 and Cinacalcet, which are known as calcimimetic agents. ${ }^{[1]}$ To the best of our knowledge, although results of excellent previous works have been reported by several groups, there is no example of application to the synthesis of such useful compounds by asymmetric arylation of aliphatic aldimines.

\section{Results and Discussion}

We began our study by examining reaction parameters such as catalyst precursors and ligands in asymmetric arylation of aliphatic aldimine 1a as a model substrate with phenyl boronic acid (Table 1). The use of cationic rhodium ([Rh(nbd) $\left.\left.)_{2}\right]\left(\mathrm{BF}_{4}\right)\right)$ and $\mathrm{N}$-Me-BIPAM proved to be particularly effective in terms of yields compared with other precursors and ligands $(73 \%, 99 \%$ ee entry 2). Precursor screening showed that the counter anion of the cationic rhodium complex had some influence on both yield and enantioselectivity (entries 1-5). Concurrently, a neutral complex displayed somewhat low catalytic activity (entry 6). Raising the reaction temperature from 50 to $80{ }^{\circ} \mathrm{C}$ slightly increased the yield (81\% yield, 98\% ee, entry 7 ). We then examined the effects of linker atoms of BIPAM ligands, and only a moderate yield was obtained in the case of Me-BIPAM or SMe-BIPAM (entries 8 and 9). Other typical asymmetric ligands, such as BINAP or Monophos, gave the corresponding product in low yields (entries 10 and 11). Unfortunately, in our reaction conditions, aldimine 1a decomposed to give an alcohol as a byproduct by arylation of aldehydes in all cases. 
Table 1. Optimization of the reaction conditions. ${ }^{[a]}$

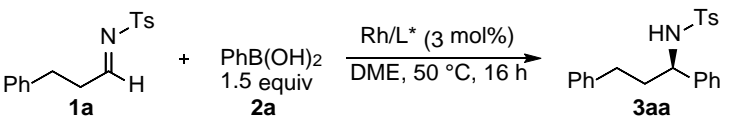

\begin{tabular}{|c|c|c|c|c|}
\hline entry & precursor & $L^{*}$ & yield (\%) & ee $(\%)$ \\
\hline 1 & {$\left[\mathrm{Rh}(\mathrm{nbd})_{2}\right]\left(\mathrm{ClO}_{4}\right)$} & $(R, R)$-N-Me-BIPAM & 69 & 98 \\
\hline 2 & {$\left[\mathrm{Rh}(\mathrm{nbd})_{2}\right]\left(\mathrm{BF}_{4}\right)$} & $(R, R)$-N-Me-BIPAM & 73 & 99 \\
\hline 3 & {$\left[\mathrm{Rh}(\mathrm{nbd})_{2}\right]\left(\mathrm{PF}_{6}\right)$} & $(R, R)$-N-Me-BIPAM & 71 & 99 \\
\hline 4 & {$\left[\mathrm{Rh}(\mathrm{nbd})_{2}\right]\left(\mathrm{SbF}_{6}\right)$} & $(R, R)-\mathrm{N}-\mathrm{Me}-\mathrm{BIPAM}$ & 71 & 97 \\
\hline 5 & {$\left[\mathrm{Rh}(\mathrm{nbd})_{2}\right]\left(\mathrm{NTf}_{2}\right)$} & $(R, R)$-N-Me-BIPAM & 66 & 98 \\
\hline 6 & $\operatorname{Rh}(\operatorname{acac})(\mathrm{coe})_{2}$ & $(R, R)$-N-Me-BIPAM & 60 & 97 \\
\hline $7^{[\mathrm{b}]}$ & {$\left[R h(n b d)_{2}\right]\left(B_{4}\right)$} & $(R, R)$-N-Me-BIPAM & 81 & 98 \\
\hline 8 & {$\left[\mathrm{Rh}(\mathrm{nbd})_{2}\right]\left(\mathrm{BF}_{4}\right)$} & $(R, R)$-Me-BIPAM & 48 & 68 \\
\hline 9 & {$\left[\mathrm{Rh}(\mathrm{nbd})_{2}\right]\left(\mathrm{BF}_{4}\right)$} & $(R, R)$-S-Me-BIPAM & 45 & 86 \\
\hline 10 & {$\left[\mathrm{Rh}(\mathrm{nbd})_{2}\right]\left(\mathrm{BF}_{4}\right)$} & $(R)$-BINAP & n.r. & - \\
\hline 11 & {$\left[\mathrm{Rh}(\mathrm{nbd})_{2}\right]\left(\mathrm{BF}_{4}\right)$} & (R)-Monophos & 28 & 7 \\
\hline
\end{tabular}

[a] Reaction conditions: $1 \mathrm{a}(0.5 \mathrm{mmol}), 2 \mathrm{a}$ (1.5 equiv), rhodium cat. (3 mol\%), and $(R, R)$-N-Me-BIPAM (1.1 equiv to Rh) in DME $(2 \mathrm{~mL})$ was stirred for $16 \mathrm{~h}$ at $50{ }^{\circ} \mathrm{C}$. [b] Reaction was conducted at $80^{\circ} \mathrm{C}$.

We also examined arylation of substrates bearing various protecting groups on the nitrogen of imine (Table 2). The type of protecting group of imines noticeably affected the reactivity, and no reaction occurred when $\mathrm{N}-\mathrm{Bn}$ (1d), N-Boc (1e), and N-OBn (1f) aldimines were used as substrates because of the instability or low electrophilicity of imines. According to the results of screening, N-sulfonyl-type aldimines such as Ts (1) and Ns (1c) are most effective in our developed catalytic arylation.

Table 2. Optimization of the protecting group of imines. ${ }^{[a]}$

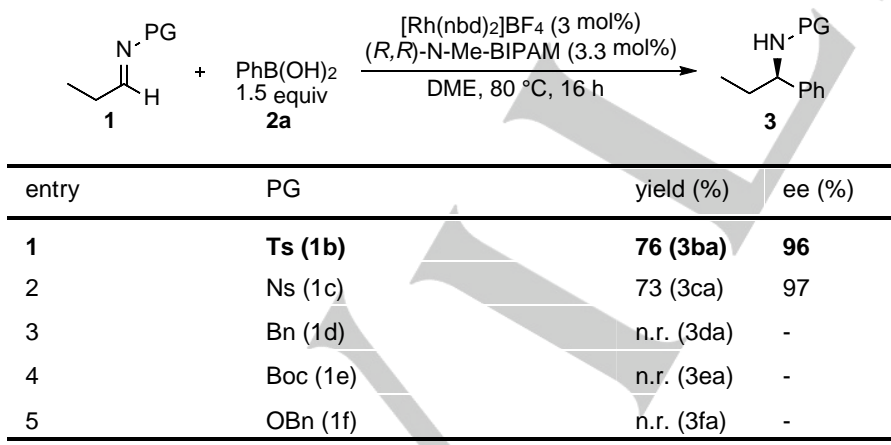

[a] Reaction conditions: 1 (0.5 mmol), 2 a (1.5 equiv), rhodium cat. (3 mol\%), and $(R, R)$-N-Me-BIPAM (1.1 equiv to $\mathrm{Rh}$ ) in DME $(2 \mathrm{~mL})$ was stirred for $16 \mathrm{~h}$ at $80^{\circ} \mathrm{C}$.

With the optimized reaction conditions in hand, various aldimine substrates and boronic acids were examined (Table 3 ). The reaction of the simplest substrate $\mathbf{1 g}$ showed a high enantioselectivity to give $\mathbf{3 g a}$ in $58 \%$ yield with $96 \%$ ee, a high enantioselectivity that has never been achieved by catalytic arylation (entry 3). Although some catalytic systems are known to promote asymmetric arylation of alkyl aldimines, there is only one report for the arylation of $\mathbf{1 9}$ with insufficient results, ${ }^{[5 d]}$ despite their structural potential including the NPS R-568 and Cinacalcet. When the $\mathrm{R}$ group was changed from methyl to other primary alkyl groups, the reactions also proceeded smoothly to afford the corresponding chiral products in good yields and high enantioselectivities (entries 4 and 5). Substrates possessing a substituent at the $\alpha$-position effectively underwent arylation to give products with high enantioselectivities (entries 6-8). In the case of these imines, Ns-aldimine was more suitable than Ts-aldimine (entries 7 and 8). Next, asymmetric arylation of1a with various arylboronic acids was examined under optimized conditions (entries 9-23). Arylboronic acid having an

Table 3. Substrate scope. ${ }^{[a]}$

\begin{tabular}{|c|c|c|c|c|c|}
\hline & 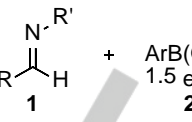 & & $\begin{array}{r}{[\mathrm{Rh}(\mathrm{nbd}) 2] \mathrm{BF}_{4}(3} \\
(R, R)-\mathrm{N}-\mathrm{Me}-\mathrm{BIPAM}(3 \\
\mathrm{DME}, 80^{\circ} \mathrm{C}, 16\end{array}$ & ${ }_{3}^{\mathrm{HN}}$ & \\
\hline entr & $\mathrm{R}$ & $\mathrm{R}^{\prime}$ & $\mathrm{Ar}$ & $\begin{array}{l}\text { yield } \\
\text { (\%) }\end{array}$ & $\begin{array}{l}\text { ee } \\
\text { (\%) }\end{array}$ \\
\hline 1 & $\mathrm{PhCH}_{2} \mathrm{CH}_{2}$ (1a) & Ts & $\mathrm{C}_{6} \mathrm{H}_{5}(\mathbf{2 a})$ & 81 (3aa) & 98 \\
\hline 2 & Et (1) & Ts & $\mathrm{C}_{6} \mathrm{H}_{5}(\mathbf{2 a})$ & 76 (3ba) & 96 \\
\hline 3 & $\operatorname{Me}(\mathbf{1 g})$ & Ts & $\mathrm{C}_{6} \mathrm{H}_{5}(\mathbf{2 a})$ & 58 (3ga) & 96 \\
\hline 4 & $n$-Pent (1h) & Ts & $\mathrm{C}_{6} \mathrm{H}_{5}(\mathbf{2 a})$ & 63 (3ha) & 95 \\
\hline 5 & $i$-Bu (1i) & Ts & $\mathrm{C}_{6} \mathrm{H}_{5}(\mathbf{2 a})$ & 74 (3ia) & 97 \\
\hline $6^{[\mathrm{b}]}$ & $i-\operatorname{Pr}(\mathbf{1} \mathbf{j})$ & Ts & $\mathrm{C}_{6} \mathrm{H}_{5}(\mathbf{2 a})$ & 66 (3ja) & 94 \\
\hline 7 & $i-\operatorname{Pr}(\mathbf{1 k})$ & Ns & $\mathrm{C}_{6} \mathrm{H}_{5}(\mathbf{2 a})$ & 79 (3ka) & 97 \\
\hline 8 & Cy (1I) & Ns & $\mathrm{C}_{6} \mathrm{H}_{5}(\mathbf{2 a})$ & 77 (3la) & 95 \\
\hline 9 & $\mathrm{PhCH}_{2} \mathrm{CH}_{2}$ (1a) & Ts & $4-\mathrm{MeOC}_{6} \mathrm{H}_{4}(\mathbf{2 b})$ & 79 (3ab) & 93 \\
\hline 10 & $\mathrm{PhCH}_{2} \mathrm{CH}_{2}(\mathbf{1} \mathbf{a})$ & Ts & $4-\mathrm{PhOC}_{6} \mathrm{H}_{4}(2 \mathrm{c})$ & 81 (3ac) & 89 \\
\hline 11 & $\mathrm{PhCH}_{2} \mathrm{CH}_{2}(\mathbf{1 a})$ & Ts & $4-\mathrm{MeSC}_{6} \mathrm{H}_{4}(2 \mathrm{~d})$ & $66(3 a d)$ & 96 \\
\hline 12 & $\mathrm{PhCH}_{2} \mathrm{CH}_{2}(\mathbf{1} \mathbf{a})$ & Ts & $4-\mathrm{MeC}_{6} \mathrm{H}_{4}(\mathbf{2 e})$ & 70 (3ae) & 97 \\
\hline 13 & $\mathrm{PhCH}_{2} \mathrm{CH}_{2}$ (1a) & Ts & $4-\mathrm{HOC}_{6} \mathrm{H}_{4}(\mathbf{2 f})$ & 61 (3af) & 89 \\
\hline 14 & $\mathrm{PhCH}_{2} \mathrm{CH}_{2}(\mathbf{1 a})$ & Ts & $4-\mathrm{PhC}_{6} \mathrm{H}_{4}(\mathbf{2 g})$ & 74 (3ag) & 86 \\
\hline 15 & $\mathrm{PhCH}_{2} \mathrm{CH}_{2}(\mathbf{1} \mathbf{a})$ & Ts & $4-\mathrm{ClC}_{6} \mathrm{H}_{4}(2 \mathrm{~h})$ & $56\left(57^{[c]}\right)(3 a h)$ & $69\left(97^{[c]}\right)$ \\
\hline $16^{[c]}$ & $\mathrm{PhCH}_{2} \mathrm{CH}_{2}(\mathbf{1 a})$ & Ts & $4-\mathrm{BrC}_{6} \mathrm{H}_{4}(\mathbf{2 i})$ & 59 (3ai) & 97 \\
\hline $17^{[\mathrm{c}]}$ & $\mathrm{PhCH}_{2} \mathrm{CH}_{2}(\mathbf{1 a})$ & Ts & $4-\mathrm{FC}_{6} \mathrm{H}_{4}(2 \mathrm{j})$ & 49 (3aj) & 96 \\
\hline $18^{[\mathrm{c}]}$ & $\mathrm{PhCH}_{2} \mathrm{CH}_{2}$ (1a) & Ts & $4-\mathrm{CF}_{3} \mathrm{OC}_{6} \mathrm{H}_{4}(\mathbf{2 k})$ & 65 (3ak) & 92 \\
\hline $19^{[c]}$ & $\mathrm{PhCH}_{2} \mathrm{CH}_{2}(\mathbf{1} \mathbf{a})$ & Ts & $4-\mathrm{CF}_{3} \mathrm{C}_{6} \mathrm{H}_{4}(2 \mathrm{I})$ & 47 (3al) & 92 \\
\hline 20 & $\mathrm{PhCH}_{2} \mathrm{CH}_{2}(\mathbf{1} \mathbf{a})$ & Ts & $3-\mathrm{MeOC}_{6} \mathrm{H}_{4}(2 \mathrm{~m})$ & 74 (3am) & 97 \\
\hline 21 & $\mathrm{PhCH}_{2} \mathrm{CH}_{2}$ (1a) & Ts & $2-\mathrm{MeOC}_{6} \mathrm{H}_{4}(\mathbf{2 n})$ & 59 (3an) & 78 \\
\hline 22 & $\mathrm{PhCH}_{2} \mathrm{CH}_{2}(\mathbf{1 a})$ & Ts & 2-naphthyl (2o) & 76 (3ao) & 88 \\
\hline 23 & $\mathrm{PhCH}_{2} \mathrm{CH}_{2}(\mathbf{1} \mathbf{a})$ & Ts & $3,5-\mathrm{MeOC}_{6} \mathrm{H}_{3}(\mathbf{2 p})$ & 70 (3ар) & 99 \\
\hline
\end{tabular}

[a] Reaction conditions: 1 (0.5 mmol), 2 (1.5 equiv), rhodium cat. (3 mol\%), and $(R, R)$-N-Me-BIPAM (1.1 equiv to Rh) in DME $(2 \mathrm{~mL})$ was stirred for $16 \mathrm{~h}$ at $80{ }^{\circ} \mathrm{C}$. [b] Reaction was conducted at $50{ }^{\circ} \mathrm{C}$. [c] Rh(acac)(coe) $)_{2}(3 \mathrm{~mol} \%)$ was used instead of $\left[\mathrm{Rh}(\mathrm{nbd})_{2}\right] \mathrm{BF}_{4}$ 
electron donating group could be effectively added to imine 1a with good to high enantioselectivities (entries 9-13). It is noteworthy that a non-protected hydroxyl group did not affect the reaction (entry 13). However, a strongly electron-deficient substituent such as a $\mathrm{Cl}, \mathrm{Br}, \mathrm{F}, \mathrm{OCF}_{3}$ or $\mathrm{CF}_{3}$ group reduced the reactivity of the substrate (entries 15-19). In these reactions, $\mathrm{Rh}(\mathrm{acac})(\mathrm{coe})_{2}$ showed higher enantioselectivities than $\left[\mathrm{Rh}(\mathrm{nbd})_{2}\right]\left(\mathrm{BF}_{4}\right)$. Arylboronic acids $\mathbf{2} \mathbf{m}-\mathbf{2} \mathbf{p}$ were also used in this reaction, giving the corresponding amines (entries 20-23). This method was successfully applied to ketimine; saccharin-derived substrate $\mathbf{4}$ afforded the corresponding product $\mathbf{5}$ in $80 \%$ yield with $>99 \%$ ee (Scheme 1 ).<smiles>CC1=NS(=O)(=O)c2ccccc21</smiles>

4

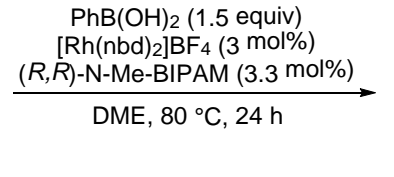

$\rightarrow$

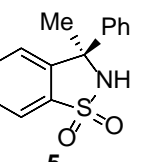

5

$80 \%,>99 \%$ ee
Scheme 1. Asymmetric arylation of ketimine 4

To demonstrate the usefulness of this method, we applied it to a synthetic key intermediates of NPS R-568 and Cinacalcet (Scheme 2). Asymmetric arylation of Ns-aldimine 6 gave the corresponding products 7 and 9 in $68 \%$ yield with $98 \%$ ee and $64 \%$ yield with $79 \%$ ee, respectively. Then the nosyl group was removed in basic conditions to give $\mathbf{8}$ in 90\% yield.

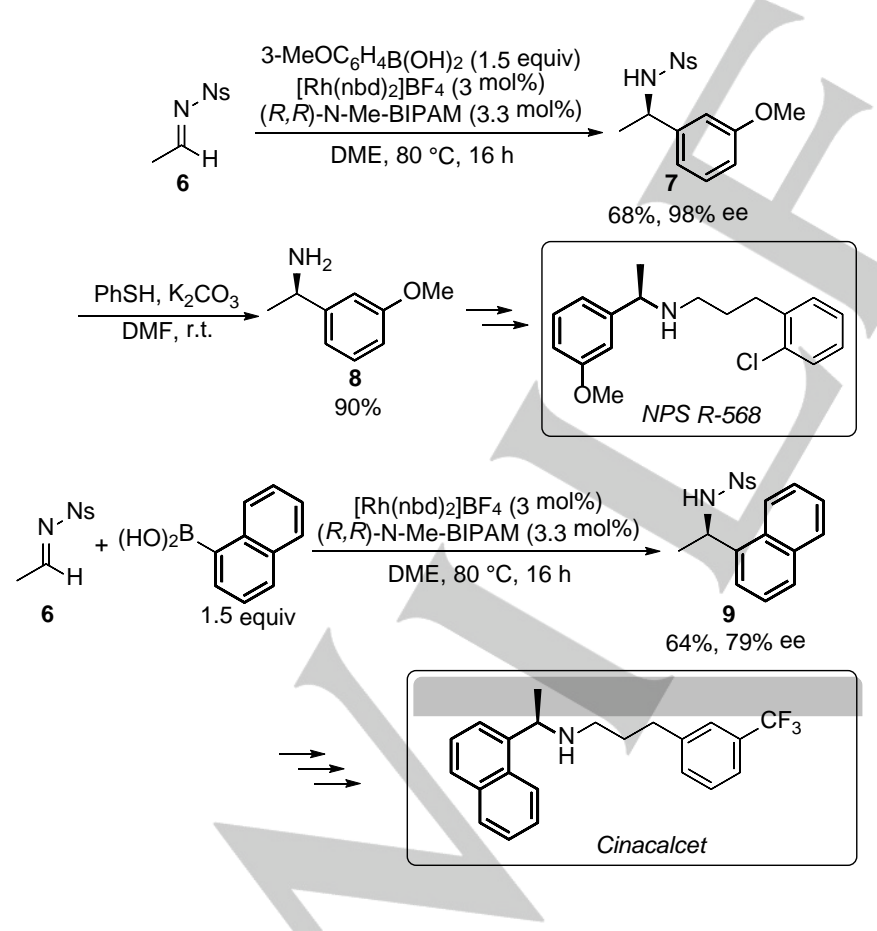

Scheme 2. Synthetic application of asymmetric arylation.
In summary, we have developed a rhodium/N-Me-BIPAMcatalyzed asymmetric arylation of aliphatic imines with arylboronic acids, which can serve as an efficient method for the synthesis of chiral $\alpha$-branched amines. We have also shown the synthetic utility on this reaction by synthesizing drug intermediates. Further studies of this catalytic transformation with the aim of expanding the substrate scope and obtaining mechanistic insights into the enantioselection are in progress.

\section{Experimental Section}

General procedure: a flame-dried flask was charged with $\left[\mathrm{Rh}(\mathrm{nbd})_{2}\right]\left(\mathrm{BF}_{4}\right)$ $(0.015 \mathrm{mmol}, 3 \mathrm{~mol} \%)$ and $(R, R)$-N-Me-BIPAM $(0.017 \mathrm{mmol}, 3.3 \mathrm{~mol} \%)$ under a nitrogen atmosphere. DME $(2 \mathrm{~mL})$ was added to the flask and the mixture was then stirred at room temperature for $30 \mathrm{~min}$ to prepare the catalyst. Arylboronic acid $(0.75 \mathrm{mmol})$, imine $(0.5 \mathrm{mmol})$ were then added to this catalyst solution. After being stirred for $16 \mathrm{~h}$ at $80^{\circ} \mathrm{C}$, the reaction mixture was extracted with $\mathrm{AcOEt}$, and dried over $\mathrm{MgSO}_{4}$. The mixture was purified by silica gel column chromatography (eluent: Hexane/AcOEt) to afford the pure $\alpha$-branched amine product.

\section{Acknowledgements}

This work was financially supported by the MEXT (Japan) "Strategic Molecular and Materials Chemistry through Innovative Coupling Reactions" of Hokkaido University.

Keywords: cationic rhodium• asymmetric arylation $\bullet \alpha$-branched amine $\bullet$ phosphoramidite $\bullet$ imines

[1] a) R. M. Barmore, S. R. Logan, B. C. VanWagenen, Tetrahedron Lett 1998, 39, 3451-3454; b) M. C. Hansen, S. L. Bachwald, Tetrahedron Lett. 1999, 40, 2033-2034; c) N. Yamazaki, M. Atobe, C. Kibayashi, Tetrahedron Lett. 2001, 42, 5029-5032; d) I. Fernandez, V. Valdivia, N. Khiar, J. Org. Chem. 2008, 73, 745-748; e) S. Banerjee, B. Smith, S. R. Hitchcock, Appl. Organometal. Chem. 2011, 25, 105-109.

[2] O. R. Thiel, C. Bernard, W. Tormos, A. Brewin, S. Hirotani, K Murakami, K. Saito, R. D. Larsen, M. J. Martinelli, P. J. Reider Tetrahedron Lett. 2008, 49, 13-15.

[3] J. Ahman, M. Birch, S. J. Haycock-Lewandowski, J. Long, A. Wilder Org. Process Res. Dev. 2008, 12, 1104-1113.

[4] For selected recent reviews on the asymmetric hydrogenation of imines and the reductive amination, see: a) T. C. Nugent, M. El-Shazly, Adv Synth. Catal. 2010, 352, 753-819; b) F. Foubelo, M. Yus, Chem. Rec. 2015, 15, 907-924.

For examples of asymmetric hydroamination of alkenes, see: c) $M$ Kawatsura, J. F. Hartwig, J. Am. Chem. Soc. 2000, 122, 9546-9547; d) Y. Miki, K. Hirano, T. Satoh, M. Miura, Angew. Chem. Int. Ed. 2013, 52, 10830-10834; Angew. Chem. 2013, 125, 11030-11034; e) S. Zhu, N. Niljianskul, S. L. Buchwald, J. Am. Chem. Soc. 2013, 135, 15746-15749; f) S.-L. Shi, S. L. Buchwald, Nat. Chem. 2015, 7, 38-44; g) Y. Yang, S.-L. Shi, D. Niu, P. Liu, S. L. Buchwald, Science 2015, 349, 62-66.

[5] For asymmetric arylation of aliphatic aldimines with arylboron reagent, see: a) M. Trincado, J. A. Ellman, Angew. Chem. Int. Ed. 2008, 47, 5623-5626; Angew. Chem. 2008, 120, 5705-5708; b) R. Shintani, R. Narui, Y. Tsutsumi, S. Hayashi, T. Hayashi, Chem. Commun. 2011, 47, 6123-6125; c) Z. Cui, H.-J. Yu, R.-F. Yang, W.-Y. Gao, C.-G. Feng, G.Q. Lin, J. Am. Chem. Soc. 2011, 133, 12394-12397; d) T. Beisel, G. Manolikakes, Org. Lett. 2015, 17, 3162-3165 
[6] For asymmetric arylation of aliphatic ketamine with arylboron reagent, see: a) R. Shintani, M. Takeda, T. Tsuji, T. Hayashi, J. Am. Chem. Soc 2010, 132, 13168-13169; b) H. Wang, T. Jiang, M.-H. Xu, J. Am. Chem. Soc. 2013, 135, 971-974; c) T. Nishimura, Y. Ebe, H. Fujimoto, T Hayashi, Chem. Commun. 2013, 49, 5504-5506; d) G. Yang, W. Zhang, Angew. Chem. Int. Ed. 2013, 52, 7540-7544; Angew. Chem. 2013, 125 7688-7692; e) Y.-J. Chen, Y.-H. Chen, C.-G. Feng, G.-Q. Lin, Org. Lett. 2014, 16, 3400-3403; f) H. Wang, Y. Li, M.-H. Xu, Org. Lett. 2014, 16 3962-3965; g) C. Jiang, Y. Lu, T. Hayashi, Angew. Chem. Int. Ed. 2014 53, 9936-9939; Angew. Chem. 2014, 126, 10094-10097; h) T. Jiang, Z Wang, M.-H. Xu, Org. Lett. 2015, 17, 528-531; i) M. Quan, G. Yang, F. Xie, I. D. Gridnev, W. Zhang, Org. Chem. Front. 2015, 2, 398-402; j) R. Takechi, T. Nishimura, Org. Biomol. Chem. 2015, 13, 4918-4924; k) J. Kong, M. McLaughlin, K. Belyk, R. Mondschein, Org. Lett. 2015, 17, 5520-5523.

[7] For transition-metal/Me-BIPAM-catalyzed asymmetric 1,2-addition, see: a) K. Kurihara, Y. Yamamoto, N. Miyaura, Adv. Synth. Catal. 2009, 351, 260-270; b) Y. Yamamoto, K. Kurihara, N. Miyaura, Angew. Chem. Int Ed. 2009, 48, 4414-4416; Angew. Chem. 2009, 121, 4478-4480; c) Y. Yamamoto, Y. Takahashi, K. Kurihara, N. Miyaura, Aust. J. Chem. 2011, 64, 1447-1453; d) Y. Yamamoto, T. Shirai, M. Watanabe, K.
Kurihara, N. Miyaura, Molecules 2011, 16, 5020-5034; e) Y. Yamamoto, T. Shirai, N. Miyaura, Chem. Commun. 2012, 48, 2803-2805; f) Y. Yamamoto, M. Yohda, T. Shirai, H. Ito, N. Miyaura, Chem. Asian J. 2012, 7, 2446-2449; g) M. Yohda, Y. Yamamoto, Org. Biomol. Chem. 2015, 13, 10874-10880; h) M. Yohda, Y. Yamamoto, Tetrahedron Asymmetry 2015, 26, 1430-1435.

[8] For transition-metal/Me-BIPAM-catalyzed asymmetric 1,4-addition, see: a) Y. Yamamoto, K. Kurihara, N. Sugishita, K. Oshita, D. Piao, N Miyaura, Chem. Lett. 2005, 34, 1224-1225; b) K. Kurihara, N. Sugishita, K. Oshita, D. Piao, Y. Yamamoto, N. Miyaura, J. Organomet. Chem. 2007, 692, 428-435; c) Y. Yamamoto, K. Kurihara, Y. Takahashi, N. Miyaura, Molecules 2013, 18, 14-26.

[9] For Rh/Me-BIPAM-catalyzed asymmetric hydrogenation, see: K. Kurihara, Y. Yamamoto, N. Miyaura, Tetrahedron Lett. 2009, 50, 3158-3160.

[10] For cationic Ir/Me-BIPAM-catalyzed direct asymmetric hydroarylations, see: a) T. Shirai, H. Ito, Y. Yamamoto, Angew. Chem. Int. Ed. 2014, 53 , 2658-2661; Angew. Chem. 2014, 126, 2696-2699; b) T. Shirai, Y. Yamamoto, Angew. Chem. Int. Ed. 2015, 54, 9894-9897; Angew. Chem. Int. Ed. 2015, 127, 10032-10035; c) T. Shirai, Y. Yamamoto, Organometallics 2015, 34, 3459-3463. 
Entry for the Table of Contents (Please choose one layout)

Layout 2:

\section{COMMUNICATION}

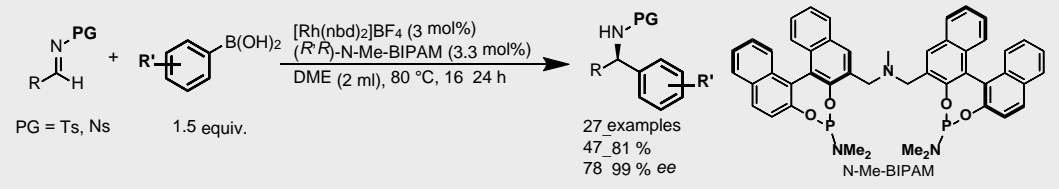

Chiral rhodium(I)/N-Me-BIPAM-catalyzed highly enantioselective arylation of aliphatic $\mathrm{N}$-sulfonyl aldimines with arylboronic acids gave the optically active $\alpha$ branched amines (up to $99 \%$ ee). In addition, this asymmetric arylation achieved efficient synthesis of (+)-NPS R-568 and Cinacalcet which are calcimimetic agents.
Naoya Kato, Tomohiko Shirai, Yasunori Yamamoto*

Page No. - Page No.

Rhodium-Catalyzed Enantioselective Arylation of Aliphatic Imines 